Dept. of Math. Univ. of Oslo

Pure Mathematics No. 20

ISSN 0806-2439 November 2006

\title{
A MAXIMUM PRINCIPLE FOR STOCHASTIC CONTROL WITH PARTIAL INFORMATION
}

\author{
FOUZIA BAGHERY ${ }^{1}$ AND BERNT ØKSENDAL ${ }^{2,3}$
}

\begin{abstract}
We study the problem of optimal control of a jump diffusion, i.e. a process which is the solution of a stochastic differential equation driven by Lévy processes. It is required that the control process is adapted to a given subfiltration of the filtration generated by the underlying Lévy processes. We prove two maximum principles (one sufficient and one necessary) for this type of partial information control. The results are applied to a partial information mean-variance portfolio selection problem in finance.
\end{abstract}

\section{INTRODUCTION}

Let $B(t)=\left(B_{1}(t), \ldots, B_{k}(t)\right)^{T}$ (where ()$^{T}$ denotes transposed) and $\eta(t)=\left(\eta_{1}(t)\right.$, $\left.\ldots, \eta_{n}(t)\right)^{T}$ be $n$-dimensional Brownian motion and $n$ independent pure jump Lévy martingales, respectively, on a filtered probability space $\left(\Omega, \mathcal{F},\left\{\mathcal{F}_{t}\right\}_{t>0}, P\right)$.

If $N_{i}(d t, d z)$ denotes the jump measure of $\eta_{i}($.$) and \nu_{i}(d z)$ denotes the Lévy measure of $\eta_{i}($.$) , then we can write$

$$
\eta_{i}(t)=\int_{0}^{t} \int_{\mathbb{R}_{0}} z \widetilde{N}_{i}(d s, d z)
$$

where

$$
\widetilde{N}_{i}(d s, d z)=N_{i}(d s, d z)-\nu_{i}(d z) d s
$$

is the compensated jump measure of $\eta_{i}(),. 1 \leq i \leq n ; \mathbb{R}_{0}=\mathbb{R}-\{0\}$.

For simplicity we assume that

$$
\int_{\mathbb{R}_{0}} z^{2} \nu_{i}(d z)<\infty \quad \text { for } i=1, \ldots, n .
$$

Suppose the state process $X(t)=X^{(u)}(t) \in \mathbb{R}^{n}$ is given by a controlled stochastic differential equation of the form

$$
\begin{aligned}
& d X(t)=b(t, X(t), u(t)) d t+\sigma(t, X(t), u(t)) d B(t) \\
& \quad+\int_{\mathbb{R}_{0}^{n}} \theta(t, X(t), u(t), z) \tilde{N}(d t, d z) ; \quad 0 \leq t \leq T \\
& X(0)=x \in \mathbb{R}^{n}
\end{aligned}
$$

Date: 26 June 2006

${ }^{1}$ Laboratoire LAMAV, Université de Valenciennes, F-59313 VALENCIENNES Cedex 9, France, Email: Fouzia.Baghery@univ-valenciennes.fr

${ }^{2}$ Center of Mathematics for Applications (CMA), University of Oslo, Box 1053 Blindern, N-0316 Oslo, Norway, Email: oksendal@math.nio.no

${ }^{3}$ Norwegian School of Economics and Business Administration, Helleveien 30, N-5045 Bergen, Norway. 
Here $b:[0, T] \times \mathbb{R}^{n} \times U \rightarrow \mathbb{R}^{n}, \sigma:[0, T] \times \mathbb{R}^{n} \times U \rightarrow \mathbb{R}^{n \times n}$ and

$\theta:[0, T] \times \mathbb{R}^{n} \times U \times \mathbb{R}_{0} \rightarrow \mathbb{R}^{n \times n}$ are given functions, $C^{1}$ with respect to $x$ and $u$, and $T>0$ is a given constant. The process $u(t)$ is our control process, required to have values in a given set $U \subset \mathbb{R}^{k}$ and required to be adapted to a given filtration $\left\{\mathcal{E}_{t}\right\}_{t \geq o}$, where

$$
\mathcal{E}_{t} \subseteq \mathcal{F}_{t}, \quad \text { for all } t
$$

For example, $\mathcal{E}_{t}$ could be the $\delta$-delayed information defined by

$$
\mathcal{E}_{t}=\mathcal{F}_{(t-\delta)^{+}} \quad ; \quad t \geq 0
$$

where $\delta>0$ is a given constant delay.

We let $\mathcal{A}=\mathcal{A}_{\mathcal{E}}$ denote a given family of $\mathcal{E}_{t}$-adapted control process

$$
u(t)=u(t, \omega):[0, T] \times \Omega \rightarrow U .
$$

Suppose we are given a performance functional

$$
J(u)=E\left[\int_{0}^{T} f(t, X(t), u(t)) d t+g(X(T))\right], \quad u \in \mathcal{A}
$$

where $f:[0, T] \times \mathbb{R}^{n} \times U \rightarrow \mathbb{R}$ and $g: \mathbb{R}^{n} \rightarrow \mathbb{R}$ are given $C^{1}$ functions satisfying the condition

$$
E\left[\int_{0}^{T}|f(t, X(t), u(t))| d t+|g(X(T))|\right]<\infty \quad ; \quad u \in \mathcal{A}
$$

The partial information control problem is to find $\Phi_{\mathcal{E}}$ and $u^{*} \in \mathcal{A}$ such that

$$
\Phi_{\mathcal{E}}=\sup _{u \in \mathcal{A}} J(u)=J\left(u^{*}\right) .
$$

We emphasize that because of the general nature of the partial information filtration $\mathcal{E}_{t}$, we cannot use dynamic programming and Hamilton-Jacobi-Bellman (HJB) equations to solve the problem. Thus our problem must be distinguished from partial observation control problems. For such problems there is already a rich literature and versions of a corresponding maximum principle have been developed by many authors. See e.g. $[1,2,5,7]$ and the references therein.

It is the purpose of this paper to prove that, in spite of the non-Markovian nature of the partial information, it is possible to establish a maximum principle for such stochastic control problems. In Section 2 we will prove a maximum principle version of sufficient type (a verification theorem) and in Section 3 we prove a (weak) version of necessary type.

Our paper is related to the paper [3], where a (sufficient) maximum principle for complete information optimal control of jump diffusions is proved.

We refer to [6] for more information about stochastic control in jump diffusion markets.

\section{A PARTIAL INFORMATION SUFFICIENT MAXIMUM PRINCIPLE}

In this section we state and prove a sufficient maximum principle for the partial information control problem (1.6).

Let $\mathcal{R}$ denote the set of functions $r:[0, T] \times \mathbb{R}_{0} \rightarrow \mathbb{R}^{n \times n}$ such that 


$$
\int_{\mathbb{R}_{0}}\left|\theta_{i j}(t, x, u, z) r_{i j}(t, z)\right| \nu_{j}(d z)<\infty \quad \text { for all } i, j, t, x
$$

Define the Hamiltonian $H:[0, T] \times \mathbb{R}^{n} \times U \times \mathbb{R}^{n} \times \mathbb{R}^{n \times n} \times \mathcal{R} \rightarrow \mathbb{R}$ by

$$
\begin{aligned}
H(t, x, u, p, q, r)= & f(t, x, u)+b^{T}(t, x, u) p+\operatorname{tr}\left(\sigma^{T}(t, x, u) q\right) \\
& +\sum_{i, j=1}^{n} \int_{\mathbb{R}_{0}} \theta_{i j}(t, x, u, z) r_{i j}(t, z) \nu_{j}(d z) .
\end{aligned}
$$

The adjoint equation in the unknown $\mathcal{F}_{t}$-predictable processes $p(t), q(t), r(t, z)$ is the following backward stochastic differential equation :

$$
\begin{aligned}
& \begin{aligned}
d p(t)=- & \nabla x \\
&
\end{aligned}(t, X(t), u(t), p(t), q(t), r(t, .)) d t+q(t) d B(t) \\
&+\int_{\mathbb{R}_{0}^{n}} r(t, z) \tilde{N}(d t, d z) ; \quad 0 \leq t \leq T \\
& p(T)=\nabla g(X(T))
\end{aligned}
$$

where $\nabla_{y} \varphi()=.\left(\frac{\partial \varphi}{\partial y_{1}}, \ldots, \frac{\partial \varphi}{\partial y_{n}}\right)^{T}$ is the gradient of $\varphi: \mathbb{R}^{n} \rightarrow \mathbb{R}$ with respect to $y=\left(y_{1}, \ldots, y_{n}\right)$.

We can now state our first main result :

Theorem 2.1 (Partial information sufficient maximum principle).

Let $\hat{u} \in \mathcal{A}$ with corresponding state process $\hat{X}(t)=X^{(\hat{u})}(t)$ and suppose there exists a solution $(\hat{p}(t), \hat{q}(t), \hat{r}(t, z))$ of the corresponding adjoint equation (2.3) and (2.4) satisfying

$$
E\left[\int_{0}^{T}\left(\widehat{X}(t)-X^{(u)}(t)\right)^{T}\left\{\hat{q} \hat{q}^{T}(t)+\int_{\mathbb{R}_{0}^{n}} r r^{T}(t, z) \nu(d z)\right\}\left(\widehat{X}(t)-X^{(u)}(t)\right) d t\right]<\infty
$$

$$
\begin{aligned}
& E\left[\int_{0}^{T} \hat{p}(t)^{T}\left\{\sigma \sigma^{T}(t, X(t), u(t))+\int_{\mathbb{R}_{0}} \theta \theta^{T}\left(t, X^{(u)}(t), u(t), z\right) \nu(d z)\right\} p(t) d t\right]<\infty \\
& \quad \text { for all } u \in \mathcal{A}
\end{aligned}
$$

and

$$
E\left[\int_{0}^{T}|\nabla u H(t, \widehat{X}(t), \hat{u}(t), \hat{p}(t), \hat{q}(t), \hat{r}(t, .))|^{2} d t\right]<\infty .
$$

Moreover, suppose that for all $t \in[0, T]$,

$$
H(t, x, u, \hat{p}(t), \hat{q}(t), \hat{r}(t, .)) \text { is concave in } x, u \text { and } g(x) \text { is concave in } x,
$$


and

(2.9) (the partial information maximum condition)

$$
\begin{aligned}
& E\left[H(t, \widehat{X}(t), \hat{u}(t), \hat{p}(t), \hat{q}(t), \hat{r}(t, .)) \mid \mathcal{E}_{t}\right] \\
& \quad=\max _{u \in U} E\left[H(t, \widehat{X}(t), u, \hat{p}(t), \hat{q}(t), \hat{r}(t, .)) \mid \mathcal{E}_{t}\right] .
\end{aligned}
$$

Then $\hat{u}(t)$ is a partial information optimal control.

Proof. Choose $u \in \mathcal{A}$ and consider $J(u)-J(\hat{u})=I_{1}+I_{2}$, where

$$
I_{1}=E\left[\int_{0}^{T}\{f(t, X(t), u(t))-f(t, \widehat{X}(t), \hat{u}(t))\} d t\right]
$$

and

$$
I_{2}=E[g(X(T))-g(\widehat{X}(T))]
$$

Note that

$$
I_{1}=I_{1,1}-I_{1,2}-I_{1,3}-I_{1,4},
$$

where

$$
\begin{aligned}
I_{1,1}=E\left[\int_{0}^{T}\{H(t, X(t), u(t), \hat{p}(t), \hat{q}(t), \hat{r}(t, .))\right. \\
-H(t, \hat{X}(t), \hat{u}(t), \hat{p}(t), \hat{q}(t), \hat{r}(t, .))\} d t] \\
I_{1,2}=E\left[\int_{0}^{T}\{b(t, X(t), u(t))-b(t, \hat{X}(t), \hat{u}(t))\}^{T} \hat{p}(t) d t\right] \\
I_{1,3}=E\left[\int_{0}^{T} t r\left[\{\sigma(t, X(t), u(t))-\sigma(t, \hat{X}(t), \hat{u}(t))\}^{T} \hat{q}(t)\right] d t\right] \\
I_{1,4}=E\left[\sum _ { i , j = 1 } ^ { n } \int _ { 0 } ^ { T } \left\{\int _ { \mathbb { R } _ { 0 } } ^ { T } \left\{\theta_{i, j}(t, X(t), u(t), z)\right.\right.\right. \\
\left.\left.-\theta_{i, j}(t, \hat{X}(t), \hat{u}(t), z)\right\} \hat{r}_{i, j}(t, z) \nu_{j}(d z) d t\right] .
\end{aligned}
$$

By concavity we have

$$
\begin{gathered}
H(t, X(t), u(t), \hat{p}(t), \hat{q}(t), \hat{r}(t, .))-H(t, \widehat{X}(t), \hat{u}(t), \hat{p}(t), \hat{q}(t), \hat{r}(t, .)) \\
\leq \nabla_{x} H(t, \widehat{X}(t), \hat{u}(t), \hat{p}(t), \hat{q}(t), \hat{r}(t, .))^{T}(X(t)-\widehat{X}(t)) \\
+\nabla_{u} H(t, \widehat{X}(t), \hat{u}(t), \hat{p}(t), \hat{q}(t), \hat{r}(t, .))^{T}(u(t)-\hat{u}(t)) .
\end{gathered}
$$


Since $u \rightarrow E\left[H(t, \widehat{X}(t), u, \hat{p}(t), \hat{q}(t), \hat{r}(t,)) \mid. \mathcal{E}_{t}\right] ; u \in U$ is maximal for $u=\hat{u}(t)$ and $u(t), \hat{u}(t)$ are $\mathcal{E}_{t}$-measurable, we get by $(2.7)$

$$
\begin{aligned}
0 & \geq \nabla_{u} E\left[H(t, \widehat{X}(t), u, \hat{p}(t), \hat{q}(t), \hat{r}(t, .)) \mid \mathcal{E}_{t}\right]_{u=\hat{u}(t)}^{T}(u(t)-\hat{u}(t)) \\
& =E\left[\nabla_{u} H(t, \widehat{X}(t), \hat{u}(t), \hat{p}(t), \hat{q}(t), \hat{r}(t, .))^{T}(u(t)-\hat{u}(t)) \mid \mathcal{E}_{t}\right] .
\end{aligned}
$$

Combining (2.3), (2.5), (2.12), (2.16) and (2.17), we obtain

$$
\begin{aligned}
I_{1,1} & \leq E\left[\int_{0}^{T} \nabla_{x} H(t, \widehat{X}(t), \hat{u}(t), \hat{p}(t), \hat{q}(t), \hat{r}(t, .))^{T}(X(t)-\widehat{X}(t)) d t\right] \\
& =-E\left[\int_{0}^{T}(X(t)-\hat{X}(t))^{T} d \hat{p}(t)\right]=:-J_{1}, \quad \text { say. }
\end{aligned}
$$

Similarly, since $g$ is concave we get, by the Itô formula,

$$
\begin{aligned}
I_{2} & =E[g(X(T))-g(\widehat{X}(T))] \leq E\left[\nabla g(\widehat{X}(T))^{T}(X(T)-\widehat{X}(T))\right] \\
& =E\left[(X(T)-\widehat{X}(T))^{T} \hat{p}(T)\right] \\
& =E\left[\int_{0}^{T}(X(t)-\widehat{X}(t))^{T}(-\nabla x H(t, \widehat{X}(t), \hat{u}(t), \hat{p}(t), \hat{q}(t), \hat{r}(t, .))) d t\right] \\
& +\int_{0}^{T} \hat{p}(t)^{T}\{b(t, X(t), u(t)-b(t, \widehat{X}(t), \hat{u}(t))\} d t \\
& +\int_{0}^{T} \operatorname{tr}\left[\left\{\sigma(t, X(t), u(t)-\sigma(t, \widehat{X}(t), \hat{u}(t))\}^{T} \hat{q}(t)\right] d t\right. \\
& \left.+\int_{0}^{T} \sum_{i, j=1}^{n} \int_{\mathbb{R}_{o}}\left\{\theta_{i j}\left(t, X(t), u(t), z_{j}\right)-\theta_{i j}\left(t, \widehat{X}(t), \hat{u}(t), z_{j}\right)\right\} \hat{r}_{i j}\left(t, z_{j}\right) \nu\left(d z_{j}\right) d t\right] \\
& =J_{1}+I_{1,2}+I_{1,3}+I_{1,4} .
\end{aligned}
$$

Adding the above, we get

$$
\begin{aligned}
J(u)-J(\hat{u}) & =I_{1}+I_{2}=I_{1,1}-I_{1,2}-I_{1,3}+I_{2} \\
& \leq-J_{1}-I_{1,2}-I_{1,3}-I_{1,4}+J_{1}+I_{1,2}+I_{1,3}+I_{1,4}=0 .
\end{aligned}
$$

Since this holds for all $u \in \mathcal{A}$, the result follows.

\section{A PARTIAL INFORMATION NECESSARY MAXIMUM PRINCIPLE}

In the previous section we proved that (under some conditions) an admissible control $\hat{u}$ satisfying the partial information maximum condition (2.9) is indeed optimal. 
We now turn to the converse question : If $\hat{u}$ is optimal, does it satisfy (2.9)? The purpose of this section is to give at least a partial confirmation that this is the case.

In addition to the assumptions in Section 2 we now assume the following :

(A1) For all $t, h$ such that $0 \leq t<t+h \leq T$, all $i=1, \ldots, k$ and all bounded $\mathcal{E}_{t}$-measurable $\alpha=\alpha(\omega)$, the control $\beta(s):=\left(0, \ldots, \beta_{i}(s), 0, \ldots, 0\right) \in U \subset \mathbb{R}^{k}$ with

belongs to $\mathcal{A}_{\mathcal{E}}$.

$$
\beta_{i}(s):=\alpha_{i} \chi_{[t, t+h]^{(s)}} \quad ; s \in[o, T]
$$

(A2) For all $u, \beta \in \mathcal{A}_{\mathcal{E}}$ with $\beta$ bounded, there exists $\delta>0$ such that $u+y \beta \in \mathcal{A}_{\mathcal{E}}$ for all $y \in(-\delta, \delta)$.

For given $u, \beta \in \mathcal{A}_{\mathcal{E}}$ with $\beta$ bounded we define the process $Y(t)=Y^{(u, \beta)}(t)$ by

$$
Y(t)=\left.\frac{d}{d y} X^{(u+y \beta)}(t)\right|_{y=0}=\left(Y_{1}(t), \ldots, Y_{n}(t)\right)^{T} .
$$

Note that $Y(0)=0$ and

$$
d Y_{i}(t)=\lambda_{i}(t) d t+\sum_{j=1}^{n} \xi_{i j}(t) d B_{j}(t)+\sum_{j=1}^{n} \int_{\mathbb{R}_{0}} \zeta_{i j}(t, z) \widetilde{N}_{j}(d t, d z),
$$

where

$$
\begin{gathered}
\lambda_{i}(t)=\nabla_{x} b_{i}(t, X(t), u(t))^{T} Y(t)+\nabla_{u} b_{i}(t, X(t), u(t))^{T} \beta(t), \\
\xi_{i j}(t)=\nabla_{x} \sigma_{i j}(t, X(t), u(t))^{T} Y(t)+\nabla_{u} \sigma_{i j}(t, X(t), u(t))^{T} \beta(t)
\end{gathered}
$$

and

$$
\zeta_{i j}(t, z)=\nabla_{x} \theta_{i j}(t, X(t), u(t))^{T} Y(t)+\nabla_{u} \theta_{i j}(t, X(t), u(t))^{T} \beta(t) .
$$

Theorem 3.1 (Partial information necessary maximum principle).

Suppose that $\hat{u} \in \mathcal{A}_{\mathcal{E}}$ is a local maximum for $J(u)$, in the sense that for all bounded $\beta \in \mathcal{A}_{\mathcal{E}}$ there exists $\delta>0$ such that $\hat{u}+y \beta \in \mathcal{A}_{\mathcal{E}}$ for all $y \in(-\delta, \delta)$ and

$$
h(y):=J(\hat{u}+y \beta), \quad y \in(-\delta, \delta)
$$

is maximal at $y=0$.

Suppose there exists a solution $\hat{p}(t), \hat{q}(t), \hat{r}(t,$.$) of the associated adjoint equation$ (2.3)-(2.4), i.e.

$$
\begin{gathered}
d \hat{p}(t)=-\nabla_{x} H(t, \widehat{X}(t), \hat{u}(t), \hat{p}(t), \hat{q}(t), \hat{r}(t, .)) d t+\hat{q}(t) d B(t) \\
\quad+\int_{\mathbb{R}_{0}^{n}} r(t, z) \widetilde{N}(d t, d z) ; 0 \leq t \leq T \\
\hat{p}(T)=\nabla g(\widehat{X}(T)), \text { where } \widehat{X}=X^{(\hat{u})} .
\end{gathered}
$$

Moreover, suppose that, if $\widehat{Y}(t)=Y^{(\hat{u}, \beta)}(t)$ and $\hat{\lambda}_{i}, \hat{\xi}_{i j}$ and $\hat{\zeta}_{i j}$ are the corresponding coefficients (see (3.2)-(3.6)), then

$$
E\left[\widehat{Y}(t)^{T}\left\{\hat{q} \hat{q}^{T}(t)+\int_{\mathbb{R}_{0}} r r^{T}(t, z) \nu(d z)\right\} \widehat{Y}(t) d t\right]<\infty
$$




$$
E\left[\int_{0}^{T} \hat{p}(t)^{T}\left\{\xi \xi^{T}(t, \widehat{X}, \hat{u}(t))+\int_{\mathbb{R}_{0}} \theta \theta^{T}(t, \hat{X}(t), \hat{u}(t), z) \nu(d z)\right\} \hat{p}(t) d t\right]<\infty
$$

Then $\hat{u}$ is a stationary point for $E\left[H \mid \mathcal{E}_{t}\right]$, in the sense that for a.a.t $\in[0, T]$ we have

$$
E\left[\nabla_{u} H(t, \widehat{X}(t), \hat{u}(t), \hat{p}(t), \hat{q}(t), \hat{r}(t, .)) \mid \mathcal{E}_{t}\right]=0
$$

Proof. Put $\widehat{X}(t)=X^{(\hat{u})}(t)$. Then with $h$ as in (3.6) we have

$$
\begin{aligned}
0= & h^{\prime}(0)=E\left[\left.\int_{0}^{T} \nabla x f(t, \widehat{X}(t), \hat{u}(t))^{T} \frac{d}{d y} X^{(\hat{u}+y \beta)}(t)\right|_{y=0} d t\right. \\
& \left.+\int_{0}^{T} \nabla u f(t, \widehat{X}(t), \hat{u}(t))^{T} \beta(t) d t+\left.\nabla g(\widehat{X}(T))^{T} \frac{d}{d y} X^{(\hat{u}+y \beta)}(T)\right|_{y=0}\right] \\
= & E\left[\int_{0}^{T} \nabla x f(t, \widehat{X}(t), \hat{u}(t))^{T} \hat{Y}(t) d t\right. \\
& \left.+\int_{0}^{T} \nabla u f(t, \widehat{X}(t), \hat{u}(t))^{T} \beta(t) d t+\nabla g(\widehat{X}(t))^{T} \hat{Y}(T)\right] .
\end{aligned}
$$

By (3.8), (3.9), (3.10) and the Itô formula,

$$
\begin{gathered}
E\left[\nabla g(\hat{X}(T))^{T} \hat{Y}(T)\right]=E\left[\hat{p}^{T}(T) \hat{Y}(T)\right] \\
=E\left[\sum _ { i = 1 } ^ { n } \int _ { 0 } ^ { T } \left\{\hat{p}_{i}(t)\left(\nabla_{x} b_{i}(t, \widehat{X}(t), \hat{u}(t))^{T} \hat{Y}(t)+\nabla_{u} b_{i}(t, \widehat{X}(t), \hat{u}(t))^{T} \beta(t)\right)\right.\right. \\
+\hat{Y}_{i}(t)(-\nabla x H(t, \hat{X}(t), \hat{u}(t), \hat{p}(t), \hat{q}(t), \hat{r}(t, .)))_{i} \\
+\sum_{j=1}^{n} \hat{q}_{i j}(t)\left(\nabla_{x} \sigma_{i j}(t, \widehat{X}(t), \hat{u}(t))^{T} \hat{Y}(t)+\nabla_{u} \sigma_{i j}(t, \widehat{X}(t), \hat{u}(t))^{T} \beta(t)\right) \\
\left.\left.+\sum_{j=1}^{n} \int_{\mathbb{R}} \hat{r}_{i j}(t, z)\left(\nabla_{x} \theta_{i j}(t, \widehat{X}(t), \hat{u}(t), z)^{T} \hat{Y}(t)+\nabla_{u} \theta_{i j}(t, \widehat{X}(t), \hat{u}(t), z)^{T} \beta(t)\right)\right\} d t\right] .
\end{gathered}
$$

Now

$$
\begin{aligned}
\nabla_{x} H(t, x, u, p, q, r) & =\nabla_{x} f(t, x, u)+\sum_{j=1}^{n} \nabla_{x} b_{j}(t, x, u) p_{j}+\sum_{k, j=1}^{n} \nabla_{x} \sigma_{k j}(t, x, u) q_{k j} \\
& +\sum_{k, j=1}^{n} \int_{\mathbb{R}_{0}} \nabla_{x} \theta_{k j}(t, x, u, z) r_{k j}(t, z) \nu_{j}(d z),
\end{aligned}
$$

and similarly with $\nabla_{u} H(t, x, u, p, q, r)$. 
Combined with (3.12) and (3.10) this gives

$$
\begin{aligned}
& 0=E\left[\int _ { 0 } ^ { T } \sum _ { i = 1 } ^ { n } \left\{\frac{\partial f}{\partial u_{i}}(t, \widehat{X}(t), \hat{u}(t))\right.\right. \\
& +\sum_{j=1}^{n}\left(\hat{p}_{j}(t) \frac{\partial b_{j}}{\partial u_{i}}(t, \widehat{X}(t), \hat{u}(t))+\sum_{k=1}^{n}\left[\hat{q}_{k j}(t) \frac{\partial \sigma_{k j}}{\partial u_{i}}(t, \widehat{X}(t), \hat{u}(t))\right.\right. \\
& \left.\left.\left.+\quad \int_{\mathbb{R}_{0}} \hat{r}_{k j}(t, z) \frac{\partial \theta_{k j}}{\partial u_{i}}(t, \widehat{X}, \hat{u}(t), z) \nu_{j}(d z)\right]\right\} \beta_{i}(t) d t\right] \\
& =\quad E\left[\int_{0}^{T} \nabla u(t, \hat{X}(t), \hat{u}(t), \hat{p}(t), \hat{q}(t), \hat{r}(t, .))^{T} \beta(t) d t\right] .
\end{aligned}
$$

Fix $t \in[0, T]$ and apply the above to $\beta=\left(0, \ldots, \beta_{i}, \ldots, 0\right)$ where

$$
\beta_{i}(s):=\alpha_{i} \chi_{[t, t+h)}(s) ; s \in[0, T]
$$

where $t+h \leq T$ and $\alpha_{i}=\alpha_{i}(\omega)$ is bounded, $\mathcal{E}_{t}$-measurable. Then (??) leads to

$$
E\left[\int_{t}^{t+h} \frac{\partial}{\partial u_{i}} H(s, \widehat{X}(s), \hat{u}(s), \hat{p}(s), \hat{q}(s), \hat{r}(s, .)) \alpha_{i} d s\right]=0
$$

Differentiating with respect to $h$ at $h=0$ gives

$$
E\left[\frac{\partial}{\partial u_{i}} H(t, \widehat{X}(t), \hat{u}(t), \hat{p}(t), \hat{q}(t), \hat{r}(t, .)) \alpha_{i}\right]=0 .
$$

Since this holds for all bounded $\mathcal{E}_{t}$-measurable $\alpha_{i}$, we conclude that using (2.7),

$$
E\left[\frac{\partial}{\partial u_{i}} H(t, \widehat{X}(t), \hat{u}(t), \hat{p}(t), \hat{q}(t), \hat{r}(t, .)) \mid \mathcal{E}_{t}\right]=0
$$

as claimed.

\section{AN APPLICATION TO FINANCE}

To illustrate our results, we give a partial information extension of Example 3.1 in [3]. See also [4] for a paper of related interest.

Suppose we have a market with the following two investment possibilities :

(i) a risk free asset, where the unit price $S_{o}(t)$ at time $t$ is given by

$$
d S_{0}(t)=\rho_{t} S_{0}(t) d t ; \quad S_{0}(0)=1
$$

(ii) a risky asset, where the unit price $S_{1}(t)$ at time $t$ is given by

$$
d S_{1}(t)=S_{1}\left(t^{-}\right)\left[\begin{array}{c}
\left.\alpha_{t} d t+\beta_{t} d B_{t}+\int_{\mathbb{R}_{o}} \gamma(t, z) \tilde{N}(d t, d z)\right] \\
S_{1}(0)>0 .
\end{array}\right.
$$

Here $\rho_{t}, \alpha_{t}, \beta_{t}$ and $\gamma(t, z)$ are bounded deterministic functions and we assume that

$$
\alpha_{t}>\rho_{t} \text { and } \Lambda_{t}:=\beta_{t}^{2}+\int_{\mathbb{R}_{o}} \gamma^{2}(t, z) \nu(d z) \geq \varepsilon>0 \quad \text { for all } t \in[0, T]
$$

for some $\varepsilon>0$, where $T>0$ is a given constant.

We assume that

$$
\gamma(t, z)>-1 \quad \text { for all } t, z
$$


This ensures that $S_{1}(t)>0$ for all $t$.

Let $\mathcal{E}_{t} \subseteq \mathcal{F}_{t}$ be a given sub-filtration.

A portfolio in this market is an $\mathcal{E}_{t}$-predictable process $w(t)=\left(u_{0}(t), u_{1}(t)\right) \in \mathbb{R}^{2}$ giving the number of units held at time $t$ of the risk free and the risky asset, respectively. The corresponding wealth process $X(t)=X^{(w)}(t)$ is defined by

$$
d X^{(w)}(t)=u_{0}(t) d S_{0}(t)+u_{1}(t) d S_{1}(t) .
$$

Let

$$
u(t)=u_{1}(t) S_{1}(t)
$$

denote the amount invested in the risky asset at time $t$ and write $X^{(u)}(t)=X^{(w)}(t)$ from now on.

We say that the portfolio $u(t)$ is $\mathcal{E}$-admissible if $u(t)$ is $\mathcal{E}_{t}$-predictable and the wealth process $\left\{X^{(u)}(t)\right\}_{t \in[0, T]}$ is lower bounded. The set of all $\mathcal{E}$-admissible portfolios is denoted by $\mathcal{A}_{\mathcal{E}}$.

If $u \in \mathcal{A}_{\mathcal{E}}$ then the corresponding wealth equation can be written

$$
\begin{aligned}
d X^{(u)}(t)=\{ & \left.\rho_{t} X^{(u)}(t)+\left(\alpha_{t}-\rho_{t}\right) u(t)\right\} d t+\beta_{t} u(t) d B(t) \\
& +\int_{\mathbb{R}} \gamma(t, z) u(t) \tilde{N}(d t, d z) ; X^{(u)}(0)=x>0 .
\end{aligned}
$$

The partial information mean-variance portfolio selection problem is to find the portfolio $\hat{u} \in \mathcal{A}_{\mathcal{E}}$ which minimizes the variance

$$
\operatorname{Var} X^{(u)}(T)=E\left[\left(X^{(u)}(T)-E\left[X^{(u)}(T)\right]\right)^{2}\right]
$$

under the constraint that

$$
E\left[X^{(u)}(T)\right]=A,
$$

where $A$ is a given constant. By means of the Lagrange multipliers method we see that the problem is equivalent to minimizing

$$
E\left[\left(X^{(u)}(T)-a\right)^{2}\right]
$$

for a given constant $a$, without constraints.

This again is equivalent to the problem to find $\hat{u} \in \mathcal{A}_{\varepsilon}$ such that

$$
\sup _{u \in \mathcal{A}_{\mathcal{E}}} E\left[-\frac{1}{2}\left(X^{(u)}(T)-a\right)^{2}\right]=E\left[-\frac{1}{2}\left(X^{(\hat{u})}(T)-a\right)^{2}\right] .
$$

To solve this we first write down the Hamiltonian

$$
H(t, x, u, p, q, r)=\left\{\rho_{t} x+\left(\alpha_{t}-\rho_{t}\right) u\right\} p+\beta_{t} u q+u \int_{\mathbb{R}_{0}} \gamma(t, z) r(t, z) \nu(d z)
$$

and the adjoint equation

$$
\left\{\begin{array}{l}
d p(t)=-\rho_{t} p(t) d t+q(t) d B(t)+\int_{\mathbb{R}_{0}} r(t, z) \tilde{N}(d t, d z) ; t \in(0, T) \\
p(T)=-X(T)+a
\end{array}\right.
$$


We follow the approach in [3] and try a solution $p(t)$ of the form

$$
p(t)=\varphi_{t} X(t)+\psi_{t},
$$

where $\varphi_{t}$ and $\psi_{t}$ are deterministic, differentiable functions. Then by the Itô formula

$$
\begin{gathered}
d p(t)=\left[\varphi_{t} \rho_{t} X(t)+\varphi_{t}\left(\alpha_{t}-\rho_{t}\right) u(t)+X(t) \varphi_{t}^{\prime}+\psi_{t}^{\prime}\right] d t \\
+\varphi_{t} \beta_{t} u(t) d B(t)+\varphi_{t} u(t) \int_{\mathbb{R}_{o}} \gamma(t, z) \widetilde{N}(d t, d z) .
\end{gathered}
$$

Comparing (4.13) and (4.14) we get

$$
\begin{gathered}
\varphi_{t} \rho_{t} X(t)+\varphi_{t}\left(\alpha_{t}-\rho_{t}\right) u(t)+X(t) \varphi_{t}^{\prime}+\psi_{t}^{\prime}=-\rho_{t}\left(\varphi_{t} X(t)+\psi_{t}\right), \\
q(t)=\varphi_{t} \beta_{t} u(t)
\end{gathered}
$$

and

$$
r(t, z)=\varphi_{t} \gamma(t, z) u(t) .
$$

Let $\hat{u}(t) \in \mathcal{A}_{\varepsilon}$ be a candidate for an optimal control and let $\widehat{X}(t), \hat{p}(t), \hat{q}(t)$ and $\hat{r}(t, z)$ be the corresponding solutions of (4.7) and (4.12).

Then

$$
\begin{aligned}
& E\left[H(t, \widehat{X}(t), u, \hat{p}(t), \hat{q}(t), \hat{r}(t, .)) \mid \mathcal{E}_{t}\right]=\rho_{t} E\left[\widehat{X}(t) \hat{p}(t) \mid \mathcal{E}_{t}\right] \\
& \quad+u E\left[\left(\alpha_{t}-\rho_{t}\right) \hat{p}(t)+\beta_{t} \hat{q}(t)+\int_{\mathbb{R}_{0}} \gamma(t, z) \hat{r}(t, z) \nu(d z) \mid \mathcal{E}_{t}\right] .
\end{aligned}
$$

Since this is a linear expression in $u$, we guess that the coefficient of $u$ must vanish, i.e.

$$
\left(\alpha_{t}-\rho_{t}\right) E\left[\hat{p}(t) \mid \mathcal{E}_{t}\right]+\beta_{t} E\left[\hat{q}(t) \mid \mathcal{E}_{t}\right]+\int_{\mathbb{R}_{0}} \gamma(t, z) E\left[\hat{r}(t, z) \mid \mathcal{E}_{t}\right] \nu(d z)=0
$$

i.e., using (4.16) and (4.17),

$$
\left(\alpha_{t}-\rho_{t}\right)\left(\varphi_{t} E\left[\widehat{X}(t) \mid \mathcal{E}_{t}\right]+\psi_{t}\right)+\hat{u}(t) \varphi_{t}\left[\beta_{t}^{2}+\int_{\mathbb{R}_{0}} \gamma^{2}(t, z) \nu(d z)\right]=0 .
$$

This gives the solution candidate

$$
\hat{u}(t)=\frac{-\left(\alpha_{t}-\rho_{t}\right)\left(\varphi_{t} E\left[\widehat{X}(t) \mid \mathcal{E}_{t}\right]+\psi_{t}\right)}{\varphi_{t} \Lambda_{t}}
$$

with $\Lambda_{t}$ given by (4.3).

As in [3] we can now proceed to determine $\varphi_{t}$ and $\psi_{t}$. The result is

$$
\begin{gathered}
\varphi_{t}=-\exp \left(\int_{t}^{T}\left\{\frac{\left(\alpha_{s}-\rho_{s}\right)^{2}}{\Lambda_{s}}-2 \rho_{s}\right\} d s\right) ; 0 \leq t \leq T \\
\psi_{t}=a \exp \left(\int_{t}^{T}\left\{\frac{\left(\alpha_{s}-\rho_{s}\right)^{2}}{\Lambda_{s}}-\rho_{s}\right\} d s\right) ; 0 \leq t \leq T .
\end{gathered}
$$

We now verify easily that $\hat{u}(t)$ given by (4.18) - (4.20) satisfies all the requirements of Theorem 2.1 and we conclude that $\hat{u}(t)$ is indeed the optimal portfolio based on the information flow $\mathcal{E}_{t}$. 
We have proved:

Theorem 4.1. The optimal portfolio $\hat{u} \in \mathcal{A}_{\mathcal{E}}$ for the partial information meanvariance portfolio selection problem (4.8)-(4.9) is given by (4.18)-(4.20).

\section{REFERENCES}

[1] A. Bensoussan (1983) Maximum principle and dynamic programming approaches of the optimal control of partially observed diffusions. Stochastics 9, 169-222.

[2] R.J. Elliott; M. Kohlmann (1989) The variational principle for optimal control of diffusions with partial information. Systems and Control Letters 12, 63-89.

[3] N. Framstad; B. Øksendal; A. Sulem (2001) Stochastic Maximum Principle for Optimal Control of Jump Diffusions and Applications to Finance. Preprint 22, University of Oslo.

[4] H. Jin; X. ZhOU. Continuous time Markowitz's problems in an incomplete market, with constrained portfolios. Manuscipt 2005.

[5] M. Kohlmann. Optimality conditions in optimal control of jump processes - extended abstract. Proceedings in Operation Research, 7 (sixth Annual Meeting, Deutsch. Gesellsch. Operation Res., Christian-Albrechts-Univ., Kiel,1977),48-57, Physica, Wizburg,1978.

[6] B. Øksendal; A. Sulem. Applied Stochastic Control of Jump Diffusions. Springer-Verlag 2005, Berlin.

[7] S. TANg (1998) The Maximum Principle for Partially Observed Optimal Control of Stochastic Differential Equations. SIAM Journal on Control and optimization, 36 n 5, 1596-1617. 